\title{
SMALL FOUR-MANIFOLDS WITHOUT NON-SINGULAR SOLUTIONS OF NORMALIZED RICCI FLOWS*
}

\author{
MASASHI ISHIDA ${ }^{\dagger}$
}

\begin{abstract}
It is known [6] that connected sums $X \# K 3 \#\left(\Sigma_{g} \times \Sigma_{h}\right) \# \ell_{1}\left(S^{1} \times S^{3}\right) \# \ell_{2} \overline{\mathbb{C} P^{2}}$ satisfy the Gromov-Hitchin-Thorpe type inequality, but can not admit non-singular solutions of the normalized Ricci flow for any initial metric, where $\Sigma_{g} \times \Sigma_{h}$ is the product of two Riemann surfaces of odd genus, $\ell_{1}, \ell_{2}>0$ are sufficiently large positive integers, $g, h>3$ are also sufficiently large positive odd integers, and $X$ is a certain irreducible symplectic 4-manifold. These exmples are closely related with a conjecture of Fang, Zhang and Zhang [10]. In the current article, we point out that there still exist 4-manifolds with the same property even if $\ell_{1}=\ell_{2}=0$ and $g=h=3$. The topology of these new examples are smaller than that of previously known examples.
\end{abstract}

Key words. Four-manifold, Ricci flow, non-singular solution.

AMS subject classifications. 53C44, 57R57, 53C25.

1. Introduction. Let $X$ be a closed oriented Riemannian manifold of dimension $n \geq 3$. The normalized Ricci flow on $X$ is the following evolution equation:

$$
\frac{\partial}{\partial t} g=-2 R i c_{g}+\frac{2}{n}\left(\frac{\int_{X} s_{g} d \mu_{g}}{v o l_{g}}\right) g
$$

where $R i c_{g}$ is the Ricci curvature of the evolving Riemannian metric $g, s_{g}$ is the scalar curvature of the evolving Riemannian metric $g, \operatorname{vol}_{g}:=\int_{X} d \mu_{g}$ and $d \mu_{g}$ is the volume measure with respect to $g$. In [19], Hamilton introduced a nice class of solutions of (1), which is so called non-singular. Recall that a solution $\{g(t)\}, t \in[0, T)$, to (1) is called non-singular if $T=\infty$ and the Riemannian curvature tensor $R m_{g(t)}$ of $g(t)$ satisfies $\sup _{X \times[0, T)}\left|R m_{g(t)}\right|<\infty$. In particular, Hamilton [19] classified non-singular solutions to the normalized Ricci flow on a closed 3-manifold. After this pioneering work of Hamilton in dimension 3, Fang, Y.G. Zhang and Z.Z. Zhang [10] studied the properties of non-singular solutions to the normalized Ricci flow in higher dimensions. In the beautiful article [10], among other things, it was proved that the existence of the non-singular solution of the normalized Ricci flow forces a constraint on the Euler characteritic $\chi(X)$ and signature $\tau(X)$ of a given 4-manifold $X$. Based on this result, they proposed the following conjecture:

Conjecture 1 (Conjecture 1.8 in $[10]$ ). Let $X$ be a closed oriented smooth Riemannian 4-manifold with $\|X\| \neq 0$ and $\bar{\lambda}(X)<0$, where $\|X\|$ is the Gromov's simplicial volume of $X$ and $\bar{\lambda}(X)$ is the Perelman's $\bar{\lambda}$ invariant. If there is a nonsingular solution to the normalized Ricci flow on $X$, then the Gromov-Hitchin-Thorpe type inequality holds:

$$
2 \chi(X)-3|\tau(X)| \geq \frac{1}{1296 \pi^{2}}\|X\|
$$

Here, the Perelman's $\bar{\lambda}$ invariant $[23,24]$ of $X$ is a differential topological invariant defined by $\bar{\lambda}(X)=\sup _{g \in \mathcal{R}_{X}} \lambda_{g}\left(v l_{g}\right)^{2 / n}$, where $\mathcal{R}_{X}$ is the space of all Riemmannian

\footnotetext{
*Received September 26, 2012; accepted for publication April 18, 2013.

$\dagger$ Department of Mathematics, Graduate School of Science, Osaka University, 1-1, Machikaneyama, Toyonaka, Osaka, 560-0043, Japan (ishida@math.sci.osaka-u.ac.jp).
} 
metrics on $X, \lambda_{g}$ is the lowest eigenvalue of the elliptic operator $4 \Delta_{g}+s_{g}$, and $\Delta=d^{*} d=-\nabla \cdot \nabla$ is the positive-spectrum Laplace-Beltrami operator associated with $g$. See also [20].

To the best of our knowledge, Conjecture 1 still remains open. However, in a joint work with Baykur [6], the present author has shown that the converse of Conjecture 1 dose not hold in general. In fact, for $\ell_{1}, \ell_{2}>0$ which are sufficiently large positive integers, and for $g, h>3$ which are also sufficiently large positive odd integers, it was proved in [6] that a connected sum of type

$$
M:=X \# K 3 \#\left(\Sigma_{g} \times \Sigma_{h}\right) \# \ell_{1}\left(S^{1} \times S^{3}\right) \# \ell_{2} \overline{\mathbb{C} P^{2}}
$$

has the following properties, where $X$ is a certain irreducible symplectic 4-manifold, $K 3$ is the $K 3$ surface, $\Sigma_{g} \times \Sigma_{h}$ is the product of two Riemann surfaces $\Sigma_{g}, \Sigma_{h}$ of odd genus and $\overline{\mathbb{C} P^{2}}$ is the complex projective plane with the reversed orientation:

1. $M$ has $\|M\| \neq 0$ and satisfies the strict case of the inequality (2):

$$
2 \chi(M)-3|\tau(M)|>\frac{1}{1296 \pi^{2}}\|M\| .
$$

2. $M$ admits infinitely many distinct smooth structures for which Perelman's $\bar{\lambda}$ invariant is negative and there is no non-singular solution to the normalized Ricci flow for any initial metric.

In what follows, we call these properties 1 and $2 \infty$-property $\mathcal{R}$ for simplicity. Notice that the existence of 4 -manifolds with $\infty$-property $\mathcal{R}$ particularly implies that the converse of Conjecture 1 does not hold in general. Namely, this tells us that the existence and non-existence of non-singular solutions are not controled by the topological information like (2). Moreover, these also provide us new examples of 4-manifold without Einstein metrics because Einstein metric is an example of non-singular solution.

However, in the construction of these examples, we took sufficiently large integers $\ell_{1}, \ell_{2}, g, h$. Therefore, it is a natural question to ask whether there still exists a 4 manifold with $\infty$-property $\mathcal{R}$ for small $\ell_{1}, \ell_{2}, g, h$. The main purpose of the current article is to give a positive answer to this question. Namely, we shall prove that there still exist 4-manifolds with $\infty$-property $\mathcal{R}$ even if $\ell_{1}=\ell_{2}=0$ and $g=h=3$. In what follows, $N_{p}$ denotes a 4 -manifold with fundamental group $\mathbb{Z}_{p}, p$ odd, which is obtained from the product $L(p, 1) \times S^{1}$ of Lens space $L(p, 1)$ and $S^{1}$ by performing a 0 -surgery along $\{p t\} \times S^{1}$. The main result of the current article is as follows:

TheOrem A. For any positive integer $0 \leq n \leq 7$, there exists an irreducible symplectic 4-manifold $X_{n}$ which is homeomoprhic to
1. $4 \mathbb{C} P^{2} \#(13+n) \overline{\mathbb{C} P^{2}} \#\left(S^{1} \times S^{3}\right)$ or
2. $3 \mathbb{C} P^{2} \#(12+n) \overline{\mathbb{C} P^{2}} \# N_{p}$ or
3. $3 \mathbb{C} P^{2} \#(12+n) \overline{\mathbb{C} P^{2}}$

and a connected sum $X_{n} \# K 3 \#\left(\Sigma_{3} \times \Sigma_{3}\right)$ has $\infty$-property $\mathcal{R}$.

Acknowledgements. The author is grateful to the Max-Plank-Institut für Mathematik in Bonn for its hospitality. This work is partially supported by the Grant-in-Aid for Scientific Research (C), Japan Society for the Promotion of Science, No. 20540090.

2. Preliminaries. In the following, for any closed 4-manifold $X, b^{+}(X)$ (resp. $\left.b^{-}(X)\right)$ denotes the dimension of a maximal linear subspace of $H^{2}(X, \mathbb{R})$ on which the cup product pairing is positive (resp. negative) definite. Notice that $b_{2}(X)=$ $b^{+}(X)+b^{-}(X)$ and $\tau(X)=b^{+}(X)-b^{-}(X)$. 
2.1. Non-vanishing theorem of $B F_{X}$. Let $X$ be a closed smooth Riemannian 4-manifold $X$ with $b^{+}(X)>1$. Recall that a $\operatorname{spin}^{c}$-structure $\mathfrak{s}$ on $X$ induces a pair of spinor bundles $S^{ \pm}$which are Hermitian vector bundles of rank 2. A Riemannian metric on $X$ and a unitary connection $A$ on the determinant line bundle $\mathcal{L}$ induce the twisted Dirac operator $\mathcal{D}_{A}: \Gamma\left(S^{+}\right) \longrightarrow \Gamma\left(S^{-}\right)$. The Seiberg-Witten monopole equations [27] over $X$ are the following non-linear partial differential equations for a unitary connection $A$ of $\mathcal{L}$ and a spinor $\phi \in \Gamma\left(S^{+}\right)$:

$$
\mathcal{D}_{A} \phi=0, F_{A}^{+}=i q(\phi),
$$

here $F_{A}^{+}$is the self-dual part of the curvature of $A$ and $q: S^{+} \rightarrow \wedge^{+}$is a certain natural real-quadratic map, where $\wedge^{+}$is the bundle of self-dual 2 -forms. In what follows, we denote the first Chern class of the complex line bundle $\mathcal{L}$ associated with $\mathfrak{s}$ by $c_{1}(\mathfrak{s})$.

An element $\mathfrak{a} \in H^{2}(X, \mathbb{Z}) /$ torsion $\subset H^{2}(X, \mathbb{R})$ is called monopole class [21, 22] of $X$ if there exists a $\operatorname{spin}^{c}$-structure $\mathfrak{s}$ with $c_{1}^{\mathbb{R}}(\mathfrak{s})=\mathfrak{a}$ which has the property that the corresponding Seiberg-Witten monopole equations have a solution for every Riemannian metric on $X$. Here $c_{1}^{\mathbb{R}}(\mathfrak{s})$ is the image of $c_{1}(\mathfrak{s})$ in $H^{2}(X, \mathbb{R})$. It is known $[22,16]$ that the set of all monopole classes of $X$ is finite.

There are several ways to detect the existence of monopole classes. For any closed oriented smooth 4-manifold $X$ with $b^{+}(X)>1$, one can define the Seiberg-Witten invariant [27] for any $\operatorname{spin}^{c}$-structure $\mathfrak{s}$ by integrating a cohomology class on the moduli space of solutions of the Seiberg-Witten monopole equations associated with $\mathfrak{s}$ :

$$
S W_{X}: \operatorname{Spin}(X) \longrightarrow \mathbb{Z}
$$

where $\operatorname{Spin}(X)$ is the set of all $\operatorname{spin}^{c}$-structures on $X$. We call the first Chern class $c_{1}(\mathfrak{s})$ Seiberg-Witten basic class of $X$ if $S W_{X}(\mathfrak{s}) \neq 0$ for a spin ${ }^{c}$-structure $\mathfrak{s}$. In particular, Seiberg-Witten basic classes are monopole classes. Moreover, there is a sophisticated refinement of the idea of the construction of the Seiberg-Witten invariant, which is due to Bauer and Furuta $[3,4,5]$. The invariant is called the stable cohomotopy Seiberg-Witten invariant and denote it by $B F_{X}$. This invariant detects the presence of a monopole class by element of a certain complicated stable cohomotopy group $\pi_{\mathbb{T}, \mathcal{U}}^{0}(\operatorname{Pic}(X)$; ind $l)$, where see [5] for the definition of the stable cohomotopy group:

$$
B F_{X}(\mathfrak{s}) \in \pi_{\mathbb{T}, \mathcal{U}}^{0}(\operatorname{Pic}(X) ; \text { ind } l)
$$

It is known [16] that the non-triviality of the stable cohomotopy Seiberg-Witten invariants implies the existence of monopole classes.

To state a non-vanishing theorem of the stable cohomotopy Seiberg-Witten invariants, we need to fix some notations. For any $\operatorname{spin}^{c}$-structure $\mathfrak{s}$ on $X$, we introduce the following quantity:

$$
\mathfrak{S}^{i j}(\mathfrak{s}):=\frac{1}{2}<c_{1}(\mathfrak{s}) \cup \mathfrak{e}_{i} \cup \mathfrak{e}_{j},[X]>,
$$

where $\mathfrak{e}_{1}, \mathfrak{e}_{2}, \cdots, \mathfrak{e}_{s}$ is a set of generators of $H^{1}(X, \mathbb{Z})$ and $s=b_{1}(X)$. Here $[X]$ is the fundamental class of $X_{i}$ and $\langle\cdot, \cdot\rangle$ is the pairing between cohomology and homology.

Definition 2 ([6]). A closed oriented smooth 4-manifold $X$ with $b^{+}(X) \geq 2$ is called BF-admissible if the following holds: 
1. There is a spinc ${ }^{c}$-structure $\mathfrak{s}$ with $S W_{X}(\mathfrak{s}) \equiv 1(\bmod 2)$ and $c_{1}^{2}(\mathfrak{s})=2 \chi(X)+$ $3 \tau(X)$

2. $b^{+}(X)-b_{1}(X) \equiv 3(\bmod 4)$.

3. $\mathfrak{S}^{i j}(\mathfrak{s}) \equiv 0(\bmod 2)$ for any $i, j$.

Then, we have

Theorem 3 ([18]). For $i=1,2,3$, let $X_{i}$ be BF-admissible, closed oriented smooth 4-manifolds. Then a connected sum $\#_{i=1}^{j} X_{i}$ has a non-trivial stable cohomotopy Seiberg-Witten invariant, where $j=2,3$.

We shall use Theorem 3 to prove Theorem A.

2.2. Irreducible BF-admissible 4-manifolds. We need to find BF-admissible 4-manifolds to prove Theorem A. For this purpose, let us recall the following nice result on the existence of irreducible symplectic 4-manifolds, where notice that it is known [12] that any simply connected minimal symplectic 4-manifold is irreducible.

Theorem 4 (Theorem A in [1]). Let $a$ and $b$ integers satisfying $2 a+3 b \geq 0$, and $a+b \equiv 0(\bmod 4)$. If, in addtion, $b \leq-2$ is satisfied. Then there exists $a$ simply connected minimal symplectic 4-manifold $X$ with $(\chi(X), \tau(X))=(a, b)$ and odd intersection form, except possibly for $(a, b)$ equal to $(7,-3),(11,-3),(13,-5)$, or $(15,-7)$.

Consider a symplectic 4-manifold with $(\chi(X), \tau(X))=(a, b)$ in Theorem 4. Since $\chi(X)=2-2 b_{1}(X)+b_{2}(X)=2+b^{+}(X)+b^{-}(X)=a$ and $\tau(X)=b^{+}(X)-b^{-}(X)=b$ hold, we have $b^{+}(X)=\alpha-1$ and $b^{-}(X)=\beta-1$, where $\alpha:=(a+b) / 2$ and $\beta:=(a-b) / 2$. Since $X$ has odd intersection form, the celebrated result of Freedman [11] tells us that $X$ is homeomorphic to

$$
(\alpha-1) \mathbb{C} P^{2} \#(\beta-1) \overline{\mathbb{C} P^{2}} .
$$

Suppose now that $b^{+}(X)=\alpha-1 \equiv 3(\bmod 4)$, i.e., $a+b \equiv 0(\bmod 8)$. Then $X$ satisfies the second condition in Definition 2, where notice that $b_{1}(X)=0$. Since $X$ is a symplectic 4-manifold with $b^{+}(X)>1$, a famous result of Taubes [25] tells us that $X$ satisfies the first condition in Definition 2. In fact, we can take a canonical $\operatorname{spin}^{c}$ structure compatible with a symplectic structure. The third condition in Definition 2 is also satisfied since we have $b_{1}(X)=0$. Hence we obtain the following existence result of $\mathrm{BF}$-admissible 4-manifolds:

Corollary 5. Let $(a, b)$ be a pair of integers satisfying $2 a+3 b \geq 0, a+b \equiv$ $0(\bmod 8)$, and $b \leq-2$ is satisfied, except possibly for $(a, b)$ equal to $(11,-3)$, $(13,-5)$, or $(15,-7)$. Set as $\alpha=(a+b) / 2$ and $\beta=(a-b) / 2$. Then, there exists a BF-admissible, irreducible symplectic 4-manifold which is homeomorphic to $(\alpha-1) \mathbb{C} P^{2} \#(\beta-1) \overline{\mathbb{C} P^{2}}$.

In the case of non-simply connected, we have a similar result as follows:

TheOREM 6 (Theorem $\mathrm{B}$ in [6]). Let $a$ and $b$ are integers satisfying $2 a+3 b \geq$ $0, a+b \equiv 0(\bmod 8)$, and $b \leq-2$ is satisfied, except possibly for $(a, b)$ equal to $(11,-3),(13,-5)$, or $(15,-7)$. Set as $\alpha=(a+b) / 2$ and $\beta=(a-b) / 2$. Then, there exists a BF-admissible, irreducible symplectic 4-manifold with fundamental group $\mathbb{Z}$ which is homeomorphic to $\alpha \mathbb{C} P^{2} \# \beta \overline{\mathbb{C} P^{2}} \#\left(S^{1} \times S^{3}\right)$ and a BF-admissible, irreducible symplectic 4-manifold with fundamental group $\mathbb{Z}_{p}$ which is homeomorphic to ( $\alpha-$ 1) $\mathbb{C} P^{2} \#(\beta-1) \overline{\mathbb{C} P^{2}} \# N_{p}$. 
2.3. Obstruction to the non-singular solutions. We also use the following result on estimates on Perelman's $\bar{\lambda}$ invariant, which was proved in [6]:

TheOrem 7. For $m=1,2,3$, let $X_{m}$ be BF-admissible 4-manifolds. And assume that $\sum_{i=1}^{j}\left(2 \chi\left(X_{i}\right)+3 \tau\left(X_{i}\right)\right)>0$, where $j=2,3$. Then, Perelman's $\bar{\lambda}$ invariant of a connected sum $Z:=\#_{i=1}^{j} X_{i}$ satisfies

$$
\bar{\lambda}(Z) \leq-4 \pi \sqrt{2 \sum_{i=1}^{j} C\left(X_{i}\right)}<0,
$$

where $C\left(X_{i}\right):=2 \chi\left(X_{i}\right)+3 \tau\left(X_{i}\right)$.

We should notice that the first non-trivial bound for Perelman's $\bar{\lambda}$ invariant of 4-manifold was proved in an interesting article [9] by using Seiberg-Witten monopole equations.

We also have the following obstruction to the existence of non-singular solution to the normalized Ricci flow, which was also proved in [6]:

Theorem 8. For $i=1,2,3$, let $X_{i}$ be BF-admissible 4-manifolds. Assume also that $\sum_{i=1}^{j}\left(2 \chi\left(X_{i}\right)+3 \tau\left(X_{i}\right)\right)>0$ is satisfied, where $j=2,3$. Then, on a connected sum $Z:=\#_{i=1}^{j} X_{i}$, there is no non-singular solution to the normalized Ricci flow for any initial metric if

$$
12(j-1)>\sum_{i=1}^{j}\left(2 \chi\left(X_{i}\right)+3 \tau\left(X_{i}\right)\right)
$$

\section{Proof of Theorem A.}

\subsection{Case 1.}

Lemma 9. For any pair $(k, \ell)$ of positive integers satisfying

$$
-7 \leq 5 k-\ell<8,5 \ell-k \geq-103
$$

the following inequalities are satisfied simultaneously:

$$
\begin{gathered}
5 \ell-k+88+8\left(2-\frac{12}{1296 \pi^{2}}\right)>0, \\
5 k-\ell+8\left(2-\frac{12}{1296 \pi^{2}}\right)>8, \\
5 k-\ell<8 .
\end{gathered}
$$

Proof. The inequality (7) is equivalent to

$$
5 k-\ell>-8\left(1-\frac{12}{1296 \pi^{2}}\right) \text {. }
$$

Since $\pi>3$ holds, we get

$$
1-\frac{12}{1296 \pi^{2}}>1-\frac{12}{1296 \cdot 3^{2}}
$$


Therefore,

$$
-8\left(1-\frac{12}{1296 \pi^{2}}\right)<-8\left(1-\frac{12}{12963^{2}}\right)<-7 .
$$

This tells us that (9) always holds if $-7 \leq 5 k-\ell$ is satisfied. Hence both (7) and (8) are satisfied under $-7 \leq 5 k-\ell<8$. Similarly, we also have

$$
-88-8\left(2-\frac{12}{1296 \pi^{2}}\right)=-96-8\left(1-\frac{12}{1296 \pi^{2}}\right)<-103 .
$$

Therefore (6) holds if $5 \ell-k \geq-103$ is satisfied.

Let $Y_{0}$ be a Kummer surface with an elliptic fibration $Y_{0} \rightarrow \mathbb{C} P^{1}$. Let $Y_{m}$ be obtained from $Y_{0}$ by performing a logarithmic transformation of order $2 m+1$ on a nonsingular fiber of $Y_{0}$. Then, $Y_{m}$ are simply connected spin manifolds with $b^{+}\left(Y_{m}\right)=3$ and $b^{-}\left(Y_{m}\right)=19$. By the Freedman classification [11], $Y_{m}$ must be homeomorphic to a $K 3$ surface. And $Y_{m}$ is a Kähler surface with $b^{+}\left(Y_{m}\right)>1$ and hence a result of Witten [27] tells us that $\pm c_{1}\left(Y_{m}\right)$ are monopole classes of $Y_{m}$ for each $m$. We have $c_{1}\left(Y_{m}\right)=2 m \mathfrak{f}$, where $\mathfrak{f}$ is Poincaré dual to the multiple fiber which is introduced by the logarithmic transformation. See also [2]. Notice also that $Y_{m}$ is a BF-admissible 4-manifold.

On the other hand, let $X_{k, \ell}$ be any 4-manifold which is homeomorphic to $k \mathbb{C} P^{2} \# \ell \overline{\mathbb{C} P^{2}} \#\left(S^{1} \times S^{3}\right)$. Then, we have $2 \chi\left(X_{k, \ell}\right)+3 \tau\left(X_{k, \ell}\right)=5 k-\ell$ and $2 \chi\left(X_{k, \ell}\right)-3 \tau\left(X_{k, \ell}\right)=5 \ell-k$. Consider the following connected sum

$$
M_{g, h}^{k, \ell}(m):=X_{k, \ell} \# Y_{m} \#\left(\Sigma_{g} \times \Sigma_{h}\right) .
$$

Then we also have

$$
\begin{gathered}
2 \chi\left(M_{g, h}^{k, \ell}(m)\right)+3 \tau\left(M_{g, h}^{k, \ell}(m)\right)=5 k-\ell+4(g-1)(h-1)-8, \\
2 \chi\left(M_{g, h}^{k, \ell}(m)\right)-3 \tau\left(M_{g, h}^{k, \ell}(m)\right)=5 \ell-k+88+4(g-1)(h-1) .
\end{gathered}
$$

LEMma 10. Consider the connected sum (11) in the case where $g=h=3$, i.e., $M_{3,3}^{k, \ell}(m)$. Then the following inequality holds if both (6) and (7) are satisfied:

$$
2 \chi\left(M_{3,3}^{k, \ell}(m)\right)-3\left|\tau\left(M_{3,3}^{k, \ell}(m)\right)\right|>\frac{1}{1296 \pi^{2}}\left\|M_{3,3}^{k, \ell}(m)\right\| \neq 0 .
$$

Similarly, the following holds if (8) is satisfied:

$$
C\left(X_{k, \ell}\right)+C\left(Y_{m}\right)+C\left(\Sigma_{3} \times \Sigma_{3}\right)<24,
$$

where $C(X):=2 \chi(X)+3 \tau(X)$ for any closed 4-manifold $X$.

Proof. Notice that we have $C\left(X_{k, \ell}\right)=5 k-\ell, C(K 3)=0$ and $C\left(\Sigma_{3} \times \Sigma_{3}\right)=$ $4 \cdot 2 \cdot 2=16$. Therefore, (15) is equivalent to $5 k-\ell+16<24$. This is nothing but (8). On the other hand, the simplicial volume of any connected sum $M_{1} \# M_{2}$ satisfies $\left\|M_{1} \# M_{2}\right\|=\left\|M_{1}\right\|+\left\|M_{2}\right\|$. See [7, 13]. It is known that [7, 13] that any simply connected manifold has vanishing simplicial volume. In particular, we have $\left\|Y_{m}\right\|=0$. It is also [13] known that the simplicial volume vanishes for any closed 
manifold whose fundamental group is amenable. Since it is known that any abelian group is amenable, we have $\left\|X_{k, \ell}\right\|=0$ because the fundamental group of $X_{k, \ell}$ is $\mathbb{Z}$. Moreover, the following result is proved in [8]:

$$
\left\|\Sigma_{h} \times \Sigma_{g}\right\|=24(g-1)(h-1) .
$$

Hence, we have $\left\|M_{3,3}^{k, \ell}(m)\right\|=\left\|X_{k, \ell}\right\|+\left\|Y_{m}\right\|+\left\|\Sigma_{3} \times \Sigma_{3}\right\|=24 \cdot 2 \cdot 2$. In particular, $\left\|M_{3,3}^{k, \ell}(m)\right\| \neq 0$. This implies

$$
\frac{1}{1296 \pi^{2}}\left\|M_{3,3}^{k, \ell}(m)\right\|=\frac{24}{1296 \pi^{2}} 4=\frac{12}{1296 \pi^{2}} 8 .
$$

By (12), we also have $2 \chi\left(M_{3,3}^{k, \ell}(m)\right)+3 \tau\left(M_{3,3}^{k, \ell}(m)\right)=5 k-\ell+4 \cdot 2 \cdot 2-8=5 k-\ell+8 \cdot 2-8$. Therefore,

$$
2 \chi\left(M_{3,3}^{k, \ell}(m)\right)+3 \tau\left(M_{3,3}^{k, \ell}(m)\right)>\frac{1}{1296 \pi^{2}}\left\|M_{3,3}^{k, \ell}(m)\right\|
$$

is equaivalent to

$$
5 k-\ell+8 \cdot 2-8>\frac{12}{1296 \pi^{2}} 8,
$$

namely,

$$
5 k-\ell+8\left(2-\frac{12}{1296 \pi^{2}}\right)>8 .
$$

Notice that this is the inequality (7). Similarly, by (13), we also have $2 \chi\left(M_{3,3}^{k, \ell}(m)\right)-$ $3 \tau\left(M_{3,3}^{k, \ell}(m)\right)=5 \ell-k+88+4 \cdot 2 \cdot 2=5 k-\ell+88+8 \cdot 2$. Hence,

$$
2 \chi\left(M_{3,3}^{k, \ell}(m)\right)-3 \tau\left(M_{3,3}^{k, \ell}(m)\right)>\frac{1}{1296 \pi^{2}}\left\|M_{3,3}^{k, \ell}(m)\right\|
$$

is equivalent to

$$
5 \ell-k+88+8 \cdot 2>\frac{12}{1296 \pi^{2}} 8
$$

namely,

$$
5 \ell-k+88+8\left(2-\frac{12}{1296 \pi^{2}}\right)>0 .
$$

This is nothing but the inequality (6). Therefore, (14) holds if both (6) and (7) are satisfied.

Theorem 6, Lemma 9 and Lemma 10 imply

Proposition 11. Let $(a, b)$ be any pair of integers satisfying

$$
a+b \equiv 0(\bmod 8), b \leq-2,0 \leq 2 a+3 b<8 .
$$

Let $k=(a+b) / 2$ and $\ell=(a-b) / 2$. Then there exits a BF-admissible, irreducible symplectic 4 -manifold $X_{k, \ell}$ with fundamental group $\mathbb{Z}$ which is homeomorphic to 
$k \mathbb{C} P^{2} \# \ell \overline{\mathbb{C} P^{2}} \#\left(S^{1} \times S^{3}\right)$. And the connected sum $M_{3,3}^{k, \ell}(m):=X_{k, \ell} \# Y_{m} \#\left(\Sigma_{3} \times \Sigma_{3}\right)$ satisfies both (14) and (15) for each $k, \ell, m$.

Proof. First of all, notice that

$$
5 k-\ell=5 \frac{(a+b)}{2}-\frac{(a-b)}{2}=2 a+3 b, 5 \ell-k=5 \frac{(a-b)}{2}-\frac{(a+b)}{2}=2 a-3 b .
$$

Therefore, the condition (5) is equivalent to

$$
-7 \leq 2 a+3 b<8,2 a-3 b \geq-103 .
$$

Then, Lemma 9 and Lemma 10 tell us that, if (17) holds, then, for any closed 4manifold $X_{k, \ell}$ which is homeomorphic to $k \mathbb{C} P^{2} \# \ell \overline{\mathbb{C} P^{2}} \#\left(S^{1} \times S^{3}\right)$, the connected sum $M_{3,3}^{k, \ell}(m)$ satisfies both (14) and (15).

On the other hand, Theorem 6 tells us that, except possibly for $(a, b)$ equal to $(11,-3),(13,-5)$, or $(15,-7)$, for any pair $(a, b)$ of integers satisfying

$$
2 a+3 b \geq 0, a+b \equiv 0(\bmod 8), b \leq-2,
$$

there exists a BF-admissible, irreducible symplectic 4-manifold $X_{k, \ell}$ which is homeomorphic to $k \mathbb{C} P^{2} \# \ell \overline{\mathbb{C} P^{2}} \#\left(S^{1} \times S^{3}\right)$. Notice that, under $2 a+3 b \geq 0$ and $b \leq-2$, $2 a-3 b \geq-103$ always holds because $2 a-3 b \geq 2 a+3 b \geq 0$. Therefore, both (17) and (18) hold if (16) is satisfied. Notice also that $2 a+3 b \geq 9$ holds for $(a, b)=(11,-3),(13,-5),(15,-7)$. The desired result now follows.

We are now in a position to prove the Case 1 in Theorem A. First of all, for any integer $0 \leq n \leq 7$, we set

$$
a=17+n, b=-9-n .
$$

In particular, we have $a+b=8$ and $2 a+3 b=7-n$. Hence we have $a+b \equiv 0(\bmod 8)$, $0 \leq 2 a+3 b=7-n<8$ and $b \leq-2$. Notice also that

$$
k=\frac{(a+b)}{2}=4, \ell=\frac{(a-b)}{2}=13+n .
$$

Then, Proposition 11 tells us that there exists a BF-admissible, irreducible symplectic 4-manifold $X_{4,13+n}$ which is homeomorphic to $4 \mathbb{C} P^{2} \#(13+n) \overline{\mathbb{C} P^{2}} \#\left(S^{1} \times S^{3}\right)$ and $M_{3,3}^{4,13+n}(m):=X_{4,13+n} \# Y_{m} \#\left(\Sigma_{3} \times \Sigma_{3}\right)$ satisfies both (14) and (15). Notice that $M_{3,3}^{4,13+n}(m)$ satisfies the strict Gromov-Hitchin-Thorpe type inequality by (14). Moreover, Theorem 8 in the case where $j=3$ tells us that there is no non-singular solution to the normalized Ricci flow on $M_{3,3}^{4,13+n}$ for any initial metric under (15), here notice that $X_{4,13+n}, Y_{m}$ and $\left(\Sigma_{3} \times \Sigma_{3}\right)$ are all BF-admissible.

On the other hand, we have $C\left(X_{4,13+n}\right)+C\left(Y_{m}\right)+C\left(\Sigma_{3} \times \Sigma_{3}\right)=5 k-\ell+0+16=$ $2 a+3 b+16=23-n>0$. Therefore, we obtain the following bound on Perelman's $\bar{\lambda}$ invariant by $(3)$ :

$$
\bar{\lambda}\left(M_{3,3}^{4,13+n}(m)\right) \leq-4 \pi \sqrt{2(23-n)}<0 .
$$

Finally, for each $n$, we shall show that the following sequence

$$
\left\{M_{3,3}^{4,13+n}(m)\right\}_{m \in \mathbb{N}}
$$


contains infinitely many diffeo types. First of all, notice that the connected sum $X_{4,13+n}$ has non-trivial stable cohomotopy Seiberg-Witten invariants by Theorem 3 . In particular, $M_{3,3}^{3,12+n}(m)$ has monopole classes which are given by

$$
\pm c_{1}\left(X_{4,13+n}\right) \pm c_{1}\left(Y_{m}\right) \pm c_{1}\left(\Sigma_{3} \times \Sigma_{3}\right)
$$

where $c_{1}(X)$ denotes the first Chern class of the canonical line bundle of a closed symplectic 4-manifold $X$ and we have $c_{1}\left(Y_{m}\right)=2 m \mathfrak{f}$. Suppose now that the sequence (20) contains only finitely many diffeomorphism types. Namely, suppose that there exists a positve integer $m_{0}$ such that $M_{3,3}^{4,13+n}\left(m_{0}\right)$ is diffemorphic to $M_{3,3}^{4,13+n}(m)$ for any integer $m \geq m_{0}$. Then, by taking $m \rightarrow \infty$, we see that the set of monopole classes of the 4-manifold $M_{3,3}^{4,13+n}\left(m_{0}\right)$ is unbounded by $(21)$. However, this is a contradiction because the set of monopole classes of any given smooth 4-manifold with $b^{+}>1$ must be finite. Therefore, the sequence (20) must contain infinitely many diffeomorphism types. For any $m$, since $M_{3,3}^{4,13+n}(m)$ is homeomorphic to $M(n):=$ $4 \mathbb{C} P^{2} \#(13+n) \overline{\mathbb{C} P^{2}} \#\left(S^{1} \times S^{3}\right) \# K 3 \#\left(\Sigma_{3} \times \Sigma_{3}\right)$, we are able to conclude that $M(n)$ has $\infty$-property $\mathcal{R}$ as desired. Case 1 in Theorem A now follows.

3.2. Case 2. In this subsection, we shall prove Case 2 in Theorem A. The strategy of the proof in this case is similar to that of Case 1.

LEMma 12. For any pair $(k, \ell)$ of positive integers satisfying

$$
-11 \leq 5 k-\ell<4,5 \ell-k \geq-107,
$$

the following conditions are satisfied simultaneously:

$$
\begin{gathered}
5 \ell-k+92+8\left(2-\frac{12}{1296 \pi^{2}}\right)>0, \\
5 k-\ell+8\left(2-\frac{12}{1296 \pi^{2}}\right)>4, \\
5 k-\ell<4 .
\end{gathered}
$$

Proof. One can check that

$$
-92-8\left(2-\frac{12}{1296 \pi^{2}}\right)<-107
$$

Hence, if $5 \ell-k \geq-107$ holds, (23) is also satisfied. Similarly, we have

$$
4-8\left(2-\frac{12}{1296 \pi^{2}}\right)<-11
$$

Hence, (24) holds if $5 k-\ell \geq-11$. This tells us that both (24) and (25) are satisfied under $-11 \leq 5 k-\ell<4$.

Let $Z_{k, \ell}$ be any 4 -manifold which is homeomorphic to $k \mathbb{C} P^{2} \# \ell \overline{\mathbb{C} P^{2}} \# N_{p}$. Then, we have

$$
2 \chi\left(Z_{k, \ell}\right)+3 \tau\left(Z_{k, \ell}\right)=5 k-\ell+4,2 \chi\left(Z_{k, \ell}\right)-3 \tau\left(Z_{k, \ell}\right)=5 \ell-k+4 .
$$


Consider the following connected sum

$$
L_{g, h}^{k, \ell}(m):=Z_{k, \ell} \# Y_{m} \#\left(\Sigma_{h} \times \Sigma_{g}\right),
$$

where $Y_{m}$ is the homotopy $K 3$ surface used in Section 3.1. Then we also have

$$
\begin{gathered}
2 \chi\left(L_{g, h}^{k, \ell}(m)\right)+3 \tau\left(L_{g, h}^{k, \ell}(m)\right)=5 k-\ell+4(g-1)(h-1)-4, \\
2 \chi\left(L_{g, h}^{k, \ell}(m)\right)-3 \tau\left(L_{g, h}^{k, \ell}(m)\right)=5 \ell-k+4(g-1)(h-1)+92 .
\end{gathered}
$$

Lemma 13. Consider the connected sum (27) in the case wher $g=h=3$, i.e., $L_{3,3}^{k, \ell}(m)$. Then the following inequality holds if both (23) and (24) are satisfied:

$$
2 \chi\left(L_{3,3}^{k, \ell}(m)\right)-3\left|\tau\left(L_{3,3}^{k, \ell}(m)\right)\right|>\frac{1}{1296 \pi^{2}}|| L_{3,3}^{k, \ell}(m) \| \neq 0 .
$$

Similarly, the following holds if (25) is satisfied:

$$
C\left(Z_{k, \ell}\right)+C\left(Y_{m}\right)+C\left(\Sigma_{3} \times \Sigma_{3}\right)<24 .
$$

Proof. We have $C\left(Z_{k, \ell}\right)=5 k-\ell+4, C\left(Y_{m}\right)=0$ and $C\left(\Sigma_{3} \times \Sigma_{3}\right)=4 \cdot 2 \cdot 2=16$. Therefore, (31) is equivalent to $5 k-\ell+4+16<24$. This is (25). On the other hand, as the proof of Lemma 10, we have $\left\|L_{3,3}^{k, \ell}(m)\right\|=\left\|Z_{k, \ell}\right\|+\left\|Y_{m}\right\|+\left\|\Sigma_{3} \times \Sigma_{3}\right\|=24 \cdot 2 \cdot 2$, where notice that $\left\|Z_{k, \ell}\right\|=0$ holds because the fundamental group of $Z_{k, \ell}$ is $\mathbb{Z}_{p}$ and hence this is amenable. From the above, we get

$$
\frac{1}{1296 \pi^{2}}\left\|L_{3,3}^{k, \ell}(m)\right\|=\frac{24}{1296 \pi^{2}} 4=\frac{12}{1296 \pi^{2}} 8 .
$$

In particular, $\left\|L_{3,3}^{k, \ell}(m)\right\| \neq 0$. By $(28)$, we also have $2 \chi\left(L_{g, h}^{k, \ell}(m)\right)+3 \tau\left(L_{g, h}^{k, \ell}(m)\right)=$ $5 k-\ell+4 \cdot 2 \cdot 2-4=5 k-\ell+8 \cdot 2-4$. Therefore,

$$
2 \chi\left(L_{3,3}^{k, \ell}(m)\right)+3 \tau\left(L_{3,3}^{k, \ell}(m)\right)>\frac{1}{1296 \pi^{2}}\left\|L_{3,3}^{k, \ell}(m)\right\|
$$

is equivalent to

$$
5 k-\ell+8 \cdot 2-4>\frac{12}{1296 \pi^{2}} 8
$$

namely,

$$
5 k-\ell+8\left(2-\frac{12}{1296 \pi^{2}}\right)>4
$$

This is the inequality (24). Similarly, by (29), we also have $2 \chi\left(L_{3,3}^{k, \ell}(m)\right)-$ $3 \tau\left(L_{3,3}^{k, \ell}(m)\right)=5 \ell-k+4 \cdot 2 \cdot 2+92=5 \ell-k+92+8 \cdot 2$. Hence,

$$
2 \chi\left(L_{3,3}^{k, \ell}(m)\right)-3 \tau\left(L_{3,3}^{k, \ell}(m)\right)>\frac{1}{1296 \pi^{2}}\left\|L_{3,3}^{k, \ell}(m)\right\|
$$


is equivalent to

$$
5 \ell-k+92+8 \cdot 2>\frac{12}{1296 \pi^{2}} 8
$$

namely,

$$
5 \ell-k+92+8\left(2-\frac{12}{1296 \pi^{2}}\right)>0 .
$$

This is nothing but (23). Therefore, (30) holds if both (23) and (24) are satisfied.

By Theorem 6, Lemma 12 and Lemma 13, we obtain

\section{Proposition 14. Let $(a, b)$ be any pair of integers satisfying}

$$
a+b \equiv 0(\bmod 8), b \leq-2,0 \leq 2 a+3 b<8 .
$$

And let

$$
k=\frac{a+b}{2}-1, \ell=\frac{a-b}{2}-1 .
$$

Then there exits a BF-admissible, irreducible symplectic 4-manifold $Z_{k, \ell}$ with fundamental group $\mathbb{Z}_{p}$ which is homeomorphic to $k \mathbb{C} P^{2} \# \ell \overline{\mathbb{C} P^{2}} \# N_{p}$, and the connected sum $L_{3,3}^{k, \ell}(m):=Z_{k, \ell} \# Y_{m} \#\left(\Sigma_{3} \times \Sigma_{3}\right)$ satisfies (30) and (31) for each $k, \ell, m$.

Proof. First of all, notice that

$$
\begin{aligned}
& 5 k-\ell=5\left(\frac{(a+b)}{2}-1\right)-\left(\frac{(a-b)}{2}-1\right)=2 a+3 b-4, \\
& 5 \ell-k=5\left(\frac{(a-b)}{2}-1\right)-\left(\frac{(a+b)}{2}-1\right)=2 a-3 b-4 .
\end{aligned}
$$

Therefore, the condition (22) is equivalent to

$$
-7 \leq 2 a+3 b<8,2 a-3 b \geq-103 .
$$

By Lemma 12 and Lemma 13, if (33) holds, for any closed 4-manifold $Z_{k, \ell}$ which is homeomorphic to $k \mathbb{C} P^{2} \# \ell \overline{\mathbb{C} P^{2}} \# N_{p}$, the connected sum $L_{3,3}^{k, \ell}(m)$ satisfies (30) and (31).

Moreover, Theorem 6 tells us that, except possibly for $(a, b)$ equal to $(11,-3)$, $(13,-5)$, or $(15,-7)$, for any pair $(a, b)$ of integers satisfying

$$
2 a+3 b \geq 0, a+b \equiv 0(\bmod 8), b \leq-2,
$$

there exists a BF-admissible, irreducible symplectic 4-manifold $Z_{k, \ell}$ which is homeomorphic to $k \mathbb{C} P^{2} \# \ell \overline{\mathbb{C} P^{2}} \# N_{p}$. Notice that $2 a-3 b \geq-103$ always holds under $2 a+3 b \geq 0$ and $b \leq-2$. Therefore, both (33) and (34) hold if (32) is satisfied. The desired result now follows, where notice that $2 a+3 b \geq 9$ holds for $(a, b)=(11,-3),(13,-5),(15,-7)$.

We prove the Case 2 of Theorem $\mathrm{A}$ as follows: For any integer $0 \leq n \leq 7$, let $a=17+n$ and $b=-9-n$. In particular, we have $a+b=8 \equiv 0(\bmod 8)$, $0 \leq 2 a+3 b=7-n<8$ and $b \leq-2$. We also have

$$
k=\frac{(a+b)}{2}-1=3, \ell=\frac{(a-b)}{2}-1=12+n .
$$


Then, Proposition 14 tells us that there exists a BF-admissible, irreducible symplectic 4 -manifold $Z_{3,12+n}$ which is homeomorphic to $3 \mathbb{C} P^{2} \#(12+n) \overline{\mathbb{C} P^{2}} \# N_{p}$, and $L_{3,3}^{3,12+n}(m):=Z_{3,12+n} \# Y(m) \#\left(\Sigma_{3} \times \Sigma_{3}\right)$ satisfies (30) and (31). The connected sum $L_{3,3}^{3,12+n}(m)$ satisfies the strict Gromov-Hitchin-Thorpe type inequality by (14). And Theorem 8 implies that we have no non-singular solution to the normalized Ricci flow on $L_{3,3}^{3,12+n}(m)$ for any initial metric under (31). On the other hand, we have $C\left(Z_{3,12+n}\right)+C\left(Y_{m}\right)+C\left(\Sigma_{3} \times \Sigma_{3}\right)=7-n+16=23-n>0$. Therefore, by (3), we obtain

$$
\bar{\lambda}\left(L_{3,3}^{3,12+n}(m)\right) \leq-4 \pi \sqrt{2(23-n)}<0 .
$$

Finally, as the proof of Case 1 above, for each $n$, we are able to show that $\left\{L_{3,3}^{3,12+n}(m)\right\}_{m \in \mathbb{N}}$ contains infinitely many diffeo types by taking $m \rightarrow \infty$. For any $m$, notice that $L_{3,3}^{3,12+n}(m)$ is homeomorphic to $L(n):=3 \mathbb{C} P^{2} \#(12+$ $n) \overline{\mathbb{C} P^{2}} \# N_{p} \# K 3 \#\left(\Sigma_{3} \times \Sigma_{3}\right)$. Therefore, we are able to conclude that $L(n)$ has $\infty$ property $\mathcal{R}$ as desired.

3.3. Case 3. Finally, we shall prove Case 3 of Theorem A. Let $P_{k, \ell}$ be any 4 -manifold which is homeomorphic to $k \mathbb{C} P^{2} \# \ell \overline{\mathbb{C} P^{2}}$. Then, we have

$$
2 \chi\left(P_{k, \ell}\right)+3 \tau\left(P_{k, \ell}\right)=5 k-\ell+4,2 \chi\left(P_{k, \ell}\right)-3 \tau\left(P_{k, \ell}\right)=5 \ell-k+4 .
$$

Notice that we have $2 \chi\left(P_{k, \ell}\right)+3 \tau\left(P_{k, \ell}\right)=2 \chi\left(Z_{k, \ell}\right)+3 \tau\left(Z_{k, \ell}\right)$ and $2 \chi\left(P_{k, \ell}\right)-3 \tau\left(P_{k, \ell}\right)=$ $2 \chi\left(Z_{k, \ell}\right)-3 \tau\left(Z_{k, \ell}\right)$ by $(26)$ and $(35)$. Consider the following connects sum

$$
G_{g, h}^{k, \ell}(m):=P_{k, \ell} \# Y_{m} \#\left(\Sigma_{h} \times \Sigma_{g}\right),
$$

where $Y_{m}$ is again the homotopy $K 3$ surface used as before. Then, by using Corollary 5 instead of Theorem 6 and using the same argument with that of Proposition 14, we are able to obtain

Proposition 15. Let $(a, b)$ be any pair of integers satisfying

$$
a+b \equiv 0(\bmod 8), b \leq-2,0 \leq 2 a+3 b<8 \text {. }
$$

Let

$$
k=\frac{a+b}{2}-1, \ell=\frac{a-b}{2}-1 .
$$

Then, there exits a BF-admissible, irreducible symplectic 4-manifold $P_{k, \ell}$ which is homeomorphic to $k \mathbb{C} P^{2} \# \ell \overline{\mathbb{C} P^{2}}$ and for each $k, \ell, m, G_{3,3}^{k, \ell}(m):=P_{k, \ell} \# Y_{m} \#\left(\Sigma_{3} \times \Sigma_{3}\right)$ satisfies

$$
\begin{gathered}
2 \chi\left(G_{3,3}^{k, \ell}(m)\right)-3\left|\tau\left(G_{3,3}^{k, \ell}(m)\right)\right|>\frac{1}{1296 \pi^{2}}\left\|G_{3,3}^{k, \ell}(m)\right\| \neq 0 \\
C\left(P_{k, \ell}\right)+C\left(Y_{m}\right)+C\left(\Sigma_{3} \times \Sigma_{3}\right)<24
\end{gathered}
$$

Case 3 in Theorem A follows easily from Proposition 15. As before, for any integer $0 \leq n \leq 7$, let $a=17+n$ and $b=-9-n$. Of course, we have $a+b \equiv 0(\bmod 8)$, $0 \leq 2 a+3 b=7-n<8$ and $b<-2, k=3$ and $\ell=12+n$. Then, Proposition 15 
implies that there exists a BF-admissible, irreducible symplectic 4-manifold $P_{3,12+n}$ which is homeomorphic to $3 \mathbb{C} P^{2} \#(12+n) \overline{\mathbb{C} P^{2}}$, and

$$
G_{3,3}^{3,12+n}(m):=P_{3,12+n} \# Y(m) \#\left(\Sigma_{3} \times \Sigma_{3}\right)
$$

satisfies (37) and (38). Hence, $G_{3,3}^{3,12+n}(m)$ satisfies the strict Gromov-Hitchin-Thorpe type inequality, and there is no non-singular solution to the normalized Ricci flow on $G_{3,3}^{3,12+n}(m)$ for any initial metric by Theorem 8 . We are also able to show the following by (3):

$$
\bar{\lambda}\left(G_{3,3}^{3,12+n}(m)\right) \leq-4 \pi \sqrt{2(23-n)}<0
$$

Finally, by considering the sequence $\left\{G_{3,3}^{3,12+n}(m)\right\}_{m \in \mathbb{N}}$, we conclude that $3 \mathbb{C} P^{2} \#(12+$ $n) \overline{\mathbb{C} P^{2}} \# K 3 \#\left(\Sigma_{3} \times \Sigma_{3}\right)$ has $\infty$-property $\mathcal{R}$ for each $n$.

\section{REFERENCES}

[1] D. Akhmedov, S. Baldridge, R. I. Baykur And B. D. Park, Simply connected minimal symplectic 4-manifolds with signature less than -1, to appear in J. Eur. Math. Soc. arXiv:0705.0778.

[2] W. P. Barth, A. M. Peters, and A. Van de Ven, Compact complex surfaces, Springer (2004).

[3] S. Bauer And M. Furuta, Stable cohomotopy refinement of Seiberg-Witten invariants: I, Invent. Math., 155 (2004), pp. 1-19.

[4] S. BAUER, Stable cohomotopy refinement of Seiberg-Witten invariants: II, Invent. Math., 155 (2004), pp. 21-40.

[5] S. Bauer, Refined Seiberg-Witten invariants. Different faces of geometry, pp. 1-46, Int. Math. Ser. (N.Y.), Kluwer/Plenum, New York, 2004.

[6] R. I. BAYKUR AND M. IshIDA, Families of 4-manifolds with nontrivial stable cohomotopy Seiberg-Witten invariants, and normalized Ricci flow, arXiv:1011.2744 (2010), to appear in Journal of Geometric Analysis.

[7] A. Besse, Einstein manifolds, Springer-Verlag (1987).

[8] M. Bucher-KARlsson, The simplicial volume of closed manifolds covered by $\mathbb{H}^{2} \times \mathbb{H}^{2}$, arXiv:math/0703587 [math.DG].

[9] F. Q. FAng And Y. G. Zhang, Perelman's $\lambda$-functional and the Seiberg-Witten equations, Front. Math. China, 2 (2007), pp. 191-210.

[10] F. Q. FAng, Y. G. Zhang, AND Z. Z. Zhang, Non-singular solutions to the normalized Ricci flow equation, Math. Ann., 340 (2008), pp. 647-674.

[11] M. H. Freedman, The topology of four-dimensional manifolds, J. Differential Geom., 17 (1982), pp. 357-453.

[12] M. D. J. Hamilton And D. Kotschick, Minimality and irreducibility of symplectic fourmanifolds, Int. Math. Res. Not. 2006, Art. ID 35032.

[13] M. Gromov, Volume and bounded cohomology, Publ. Math. I.H.E.S., 56 (1982), pp. 5-99.

[14] N. J. Hitchin, Compact four-dimensional Einstein manifolds, J. Differential Geom., 9 (1974), pp. $435-441$.

[15] M. Ishida And C. LeBrun, Spin manifolds, Einstein metrics, and differential topology, Math. Res. Lett., 9 (2002), pp. 229-240.

[16] M. Ishida And C. LeBrun, Curvature, connected sums, and Seiberg-Witten theory, Comm. Anal. Geom., 11 (2003), pp. 809-836.

[17] M. IsHidA, The normalized Ricci flow on four-manifolds and exotic smooth structures, arXiv:0807.2169 (2008).

[18] M. Ishida AND H. SASAHIRA, Stable cohomotopy Seiberg-Witten invariants of connected sums of four-manifolds with positive first Betti number, arXiv:0804.3452 (2008).

[19] R. Hamilton, Non-singular solutions of the Ricci flow on three-manifolds, Comm. Anal. Geom., 7 (1999), pp. 695-729.

[20] B. Kleiner And J. Lott, Notes on Perelman's papers, arXiv: math.DG/0605667 (2006).

[21] P. B. Kronheimer, Minimal genus in $S^{1} \times M$, Invent. Math., 135 (1999), pp. 45-61.

[22] C. LeBrun, Four-manifolds, curvature bounds, and convex geometry, arXiv: math.DG/061 1450 (2006). 
[23] G. Perelman, The entropy formula for the Ricci flow and its geometric applications, arXiv: math.DG/0211159 (2002).

[24] G. Perelman, Ricci flow with surgery on three-manifolds, arXiv: math.DG/0303109 (2003).

[25] C. H. Taubes, The Seiberg-Witten invariants and symplectic forms, Math. Res. Lett., 1 (1994), pp. 809-822.

[26] J. A. Thorpe, Some remarks on the Gauss-Bonnet formula, J. Math. Mech., 18 (1969), pp. $779-786$.

[27] E. Witten, Monopoles and four-manifolds, Math. Res. Lett., 1 (1994), pp. 809-822. 